\title{
Once-daily simeprevir in combination with pegylated-interferon and ribavirin: a new horizon in the era of direct-acting antiviral agent therapy for chronic hepatitis $\mathrm{C}$
}

\author{
Shinya Maekawa $\cdot$ Nobuyuki Enomoto
}

Received: 7 December 2013/Accepted: 10 December 2013/Published online: 21 December 2013

(C) Springer Japan 2013

Hepatitis $\mathrm{C}$ virus (HCV) is a leading cause of chronic hepatitis, liver cirrhosis, and hepatocellular carcinoma (HCC), and it is estimated that infected individuals total 185 million people worldwide. In Japan, around 2 million are infected with $\mathrm{HCV}$, and more than 20 thousand die from HCV-induced HCC annually. Though viral eradication with antiviral therapies is the most important and effective choice for decreasing HCC-related deaths induced by $\mathrm{HCV}$, complete viral eradication has been quite difficult till recently, especially in patients with genotype-1 $\mathrm{HCV}$ infection because of the low response rate to interferon (IFN)-based therapy [1, 2].

In this background, development of novel direct-acting antiviral agents (DAAs) specific for $\mathrm{HCV}$ was truly a revolutionary event. In 2011, two first-generation NS3 protease inhibitors (PIs), telaprevir and boceprevir, were firstly approved among all the DAAs for clinical use in USA and Europe for genotype-1 HCV in combination with pegylated-interferon and ribavirin (PR), while telaprevir was approved in Japan in the same 2011 period. As expected, a regimen including telaprevir in combination with pegylated-interferon and ribavirin dramatically improved the sustained viral response (SVR) rate to as high as $80 \%$ in genotype-1 HCV infection. On the other hand, telaprevir has several undesirable problems. Among all, adverse events (AEs) of anemia and skin rash are serious problems of telaprevir, and Grade 3/4 skin disorders,

This comment refers to the article available at doi:10.1007/s00535013-0875-1.

\section{S. Maekawa $(\bowtie) \cdot$ N. Enomoto}

First Department of Internal Medicine, Faculty of Medicine,

University of Yamanashi, 1110 Shimokato, Chuo,

Yamanashi 409-3898, Japan

e-mail: maekawa@yamanashi.ac.jp including Stevens-Johnson syndrome and drug rashes with eosinophilia and systemic symptoms, as well as Grade 3 anemia $(<8.0 \mathrm{~g} / \mathrm{dL})$, might occur [3, 4]. Moreover, cumbersome frequent dosing three times a day (every 7-9 h) could induce poor medication adherence. Under the circumstances, it has been quite stressful for patients as well as clinicians to introduce and monitor this telaprevir-based regimen.

Simeprevir (SMV, TMC435) is classified as a secondgeneration PI with the macrocyclic structure having an advantage in the binding affinity and specificity for NS3 protease compared to the first-generation PI with the linear structure. Due to the difference in the structure, the drugresistance profile is somewhat different from that of telaprevir. Though simeprevir shows cross-resistance with telaprevir at amino acid positions of 155 and 156, most of the resistant mutation occurs at the simeprevir-specific amino acid position of 168 [5]. Though simeprevir is effective in all viral genotypes (genotype 1-6), it has the strongest antiviral activity for genotype-1a and -1b HCV infection. In particular, low AE rate and its patient-friendly once-daily dosing are the important characters of simeprevir aside from its strong antiviral activity. In the international phase II trials of simeprevir in combination with PegIFNa-2a/RBV for treatment-naïve (PILLAR study) [6] and treatment-experienced patients (ASPIRE study) [7] for HCV genotype 1-infected patients, it was demonstrated that simeprevir was generally well tolerated and had a pharmacokinetic profile supporting once-a-day (QD) dosing resulting in high virologic response rates.

In this issue of the Journal of Gastroenterology, Hayashi et al. [8] reported the important results of the phase II Dose and duration Ranging study of Antiviral agent TMC435 in Genotype $O$ ne HCV treatment-Naïve patients (DRAGON study; TMC435-C215) evaluating once-daily simeprevir 
with pegylated-interferon and ribavirin therapy for treatment-naïve, high viral-loaded hepatitis C genotype 1-infected patients in Japan. Due to the result of previous phase I study that simeprevir plasma concentration was higher in Japanese healthy volunteers compared with Caucasian volunteers, simeprevir doses of 50/100 mg QD were selected for this study, while simeprevir doses of $150 \mathrm{mg}$ QD were selected in western countries [9]. Through investigating five treatment groups (SMV12/PR24 $50 \mathrm{mg}$, SMV12/PR24 $100 \mathrm{mg}$, SMV24/PR24 $50 \mathrm{mg}$, SMV24/PR24 $100 \mathrm{mg}$, and PR48), it was disclosed that simeprevir-combined groups all achieved high SVR rate (77-92\% compared to $46 \%$ for PR). As to the AEs, simeprevir was well tolerated, and the incidence of anemia and skin rash were similar in their frequency and their grade between all the SMV groups and the PR group. Due to low AEs, therapy discontinuation rate and ribavirin dose reduction was also similar in the SMV groups and the PR group. While an $\mathrm{AE}$ of bilirubin elevation was specific to simeprevir, and it reached to grade $3(2.6-5.0 \mathrm{mg} / \mathrm{dL})$ to 4 $(>5.0 \mathrm{mg} / \mathrm{dL})$ in four patients $(5 \%)$ leading to the discontinuation of simeprevir in these individuals, the bilirubin level returned to baseline after the end of simeprevir in those patients. Since bilirubin elevation is considered to result from the blockade of bilirubin clearance-associated OATP1B1 and MRP transporters by simeprevir [10], it is considered that the bilirubin elevation by simeprevir does not reflect deterioration of liver function.

Following the results of this phase II DRAGON study, treatment dosage of simeprevir was determined as $100 \mathrm{mg}$ QD in Japan, and successive phase III CONCERTO studies for simeprevir/pegylated-interferon/ribavirin therapy have been conducted (CONCERTO- 1 for treatment-naïve, -2 for previous null responder, -3 for previous relapser, and -4 for naïve, null responder and relapser). After the completion of those CONCERTO studies with favorable outcomes for simeprevir-based regimens, once-daily simeprevir with pegylated-interferon and ribavirin therapy for high viralloaded hepatitis $\mathrm{C}$ genotype 1-infected patients was just recently approved for clinical use in Japan.

Considering the history of HCV therapy, this new therapy of once-daily simeprevir with pegylated-interferon and ribavirin therapy is ideal in its high efficacy and low AEs. Of course, it is true that DAA combination therapies without IFN (IFN-free therapies) would appear in the near future, and that these IFN-free therapies are advantageous in that they are free from IFN-related AEs. However, in terms of DAA-resistant viral mutants, it is considered that these mutant $\mathrm{HCV}$ s generally have low replication fitness, and are sensitive to IFN. Therefore, it is speculated that IFN-based DAA therapies compared to IFN-free DAA therapies are safer in preventing the development of multidrug resistant HCVs.

Taken together, the new regimen of once-daily simeprevir with pegylated-interferon and ribavirin therapy would surely be an important milestone in the therapy for high viral-loaded hepatitis $\mathrm{C}$ genotype- 1 infected patients in the era of DAA therapy.

\section{References}

1. Kim MN, Kim BK, Han KH. Hepatocellular carcinoma in patients with chronic hepatitis $\mathrm{C}$ virus infection in the AsiaPacific region. J Gastroenterol. 2013;48(6):681-8.

2. Thomas DL. Global control of hepatitis C: where challenge meets opportunity. Nat Med. 2013;19(7):850-8.

3. Chayama K, Hayes CN, Ohishi W, Kawakami Y. Treatment of chronic hepatitis $\mathrm{C}$ virus infection in Japan: update on therapy and guidelines. J Gastroenterol. 2013;48(1):1-12.

4. Kumada H, Toyota J, Okanoue T, Chayama K, Tsubouchi H, Hayashi N. Telaprevir with peginterferon and ribavirin for treatment-naive patients chronically infected with $\mathrm{HCV}$ of genotype 1 in Japan. J Hepatol. 2013;56(1):78-84.

5. Lenz O, Verbinnen T, Lin TI, Vijgen L, Cummings MD, Lindberg $\mathrm{J}$, et al. In vitro resistance profile of the hepatitis $\mathrm{C}$ virus NS3/4A protease inhibitor TMC435. Antimicrob Agents Chemother. 2010;54(5):1878-87.

6. Fried MW, Buti M, Dore GJ, Flisiak R, Ferenci P, Jacobson I, et al. Once-daily simeprevir (TMC435) with pegylated interferon and ribavirin in treatment-naive genotype 1 hepatitis $\mathrm{C}$ : the randomized PILLAR study. Hepatology. 2013;58(6):1918-29.

7. Zeuzem S, Berg T, Gane E, Ferenci P, Foster GR, Fried MW, et al. Simeprevir increases rate of sustained virologic response among treatment-experienced patients with HCV genotype-1 infection: a phase IIb trial. Gastroenterology. 2013. doi:10.1053/j. gastro.2013.10.058.

8. Hayashi N, Seto C, Kato M, Komada Y, Goto S. Once-daily simeprevir (TMC435) with peginterferon/ribavirin for treatmentnaïve hepatitis C genotype 1-infected patients in Japan: the DRAGON study. J Gastroenterol. 2013. doi:10.1007/s00535-0130875-1.

9. Verloes R, Shishido A. Phase I safety and PK of TMC435 in healthy volunteers and safety, PK and short-term efficacy in chronic hepatitis $\mathrm{C}$ infected individuals. Kobe: Abstract O-32 presented at the Japanese Hepatology Congress; 2009. p. 4-5.

10. Huisman MT, Snoeys J, Monbaliu J, Martens M, Sekar V, Raoof A. In vitro studies investigating the mechanism of interaction between TMC435 and hepatic transporters. Poster 278 presented at the 61st Annual Meeting of the American Association for the Study of Liver Diseases (AASLD), Boston, USA, October 29November 2, 2010. 